\title{
Computer Simulation of Phase Decomposition and Reversion \\ Process in the FCC Ising Model*
}

\section{By Hiroshi OKUDA** and Kozo OSAMURA $A^{* * *}$}

\begin{abstract}
Synopsis
Structure change during reversion of phase-decomposed alloys has been investigated by using the kineiic Ising model (Kawasaki dynamics) on $f c c$ lattice, and the results have been compared with the experimental results obtained by means of synchrotron radiation small-angle scattering (SR$S A X S)$ study of Al-Zn binary alloys. From the real-space configuration of solute atoms obtained by the simulation, the structure of the system was investigated. The structure function was also calculated by Fourier transform, in order that direct comparison with SAXS experiments was possible. It was made clear that the time evolution of the structure parameters during phase decomposition agreed qualitatively with those obtained by Lebowitz et al. for a simple cubic lattice. The structure change during reversion was found to be divided into two distinct stages: The earlier one is characterized by the rapid reduction of the composition of precipitates, with almost constant volume fraction. The later one is characterized as the stage where precipitates shrink to dissolve with almost constant composition. For reversion below the coexistence curve, the coarsening process at the reversion temperature followed the reversion process. It was found that the structure change during reversion in $\mathrm{Al}-\mathrm{Zn}$ binary alloys was very similar to the one observed in the present simulation, and the further discussion based on the two-phase analysis with the small-angle $X$-ray scattering results agreed well with the present results.
\end{abstract}

Key words: computer simulation; kinetic ising model; Monte Carlo method; reversion, phase decompostion, $\mathrm{Al}-\mathrm{Zn}$ alloys; G. P. zone; twophase model; small-angle $X$-ray scattering.

\section{Introduction}

The kinetic Ising model has been one of the most useful models to inquire into the kinetics of phase transition in condensed matter. In particular, phase decompositions of binary alloys on square ${ }^{1-3)}$ and cubic $^{4-11)}$ lattices have been intensively investigated mainly by Lebowitz, Borz, Gunton, Binder and their coworkers. Concerning the kinetics of phase decomposition, the time evolution of the structure function, $S(k, t)$, and related parameters were made clear, ${ }^{2,4-7)}$ and the cluster size distribution calculated directly from the configuration has been discussed in terms of nucleation theory based on cluster kinetics. ${ }^{8-10)}$ Computer simulation using the kinetic Ising model has the advantage that we can observe the system in real space and at any time during the process. In the present study, reversion phenomena in a fcc lattice which had undergone phase decomposition were investigated. This simulation is expected to well describe the structure change during reversion of a system with some extent of temperature-dependent mutual miscibility, such as $\mathrm{Al}-\mathrm{Zn}$ binary alloys which contain G. P. zones. Experimentally, until very recently it has been very difficult to obtain detailed knowledge concerning the structure change of G. P. zones during reversion, since the whole process is completed within a very short time. Recent development of synchrotron radiation small-angle X-ray scattering (SRSAXS) measurements ${ }^{12,13)}$ enables us to aquire more detailed structural information, and suggested that two elementary processes exist in the reversion process of the alloys. The earlier one is characterized by the compositional reduction inside G. P. zone, with each volume being almost constant. The later one is the stage where G. P. zones shrink and dissolve into the matrix, with constant composition of zones. However, the effect of the concentration gradient outside G. P. zone interface made it difficult to apply the twophase model analysis to the later stage of reversion. In the present paper, structural changes in the Ising lattice have been investigated, and the results have been compared with the SR-SAXS results as shown in Fig. 1.

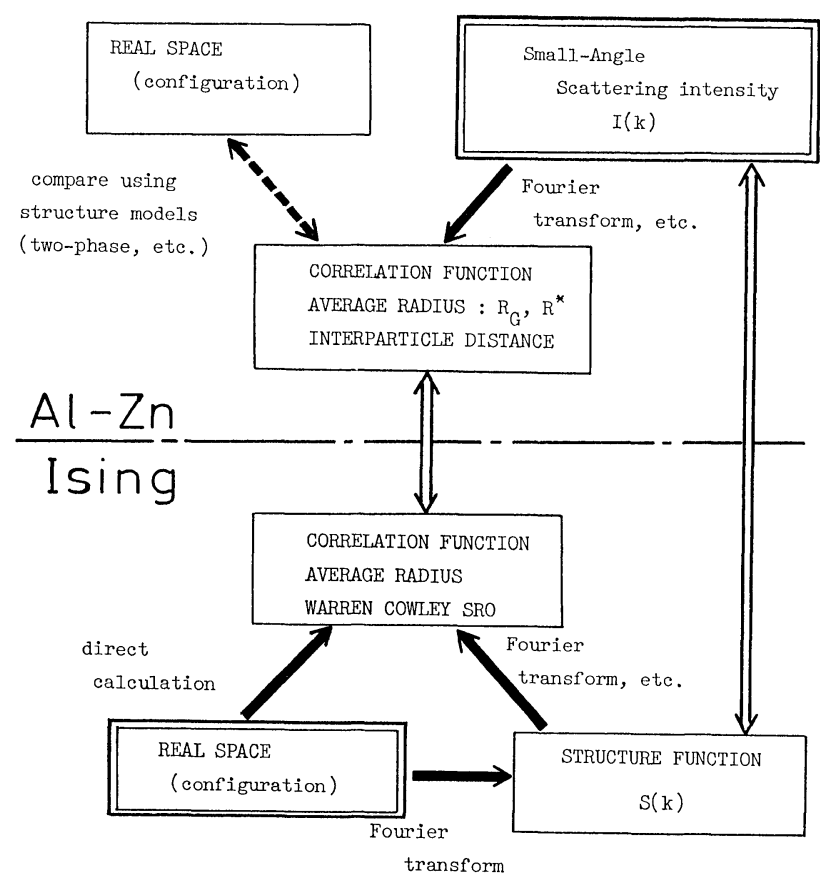

Fig. 1. Schematic drawing of the data analysis for the present simulation and SAXS experiments. Preliminarily information obtained is the atomic configuration for Ising model and the scattering intensity for SAXS experiments.

* $\quad$ Manuscript received on December 14, 1987; accepted in the final form on March 11, 1988 . (C) 1988 ISIJ

** Formerly Graduate Student, Kyoto University. Now at Fujitsu Laboratories, Atsugi, Fujitsu Laboratories, Ltd., MorinosatoWakamiya, Atsugi 243-01.

*** Department of Metallurgy, Kyoto University, Yoshida-honmanchi, Sakyo-ku, Kyoto 606. 


\section{Simulation Procedure}

The model used in the present study was the kinetic Ising model on an fcc lattice. The dimension of the system was 10, 15, 20 and 30 unit cells for each side, and periodic boundary conditions were introduced to avoid finite size effects. Temperature is measured by the unit of critical temperature, and the length is measured by the unit cell of the fcc lattice, $a$. As the time scale, Monte Carlo steps per atom (MCS) was used. Solvent and solute atom species are denoted by $A$ and $B$, respectively, and assigned to be $s(\boldsymbol{r})=-1$ and $s(\boldsymbol{r})=+1$ at each lattice site, $\boldsymbol{r}$. Solute concentrations, $C_{B}$, examined here were $7.5,10,20$ and $25 \%$. The system was solution treated and subsequently aged to form precipitates, and then reverted at various temperatures. Solution treatment, ageing and reversion were achieved by spin-exchange mechanism (Kawasaki dynamics) according to the transition probability ${ }^{14,15)}$ :

$$
W_{i j}=\tau^{-1} \exp \left(-\frac{\delta H}{k T}\right) /\left(1+\exp \left(-\frac{\delta H}{k T}\right)\right)
$$

where, $i, j: \quad$ the states before and after the exchange $\delta H:$ the energy difference between the states, which is calculated by

$$
\delta H=\delta \sum_{i} J s(\boldsymbol{r}) s\left(\boldsymbol{r}_{i}\right)
$$

The sum in Eq. (2) is taken for the nearest neighbour sites, and the exchange constant, $J$, is related to the interaction parameter appearing in the regular solution model by ${ }^{16)}$

$$
\Omega=2 J z \mathcal{N}
$$

The prefactor, $\tau$, contains the frequency term, and related to the diffusion coefficient of real alloys such as $\mathrm{Al}-\mathrm{Zn}$ alloys. The general flow chart of the present simulation is shown in Fig. 2. As shown in the figure, the system was aged at $0.5 T_{c}$ for about $10^{3} \mathrm{MCS}$ after solution treatment at $3.0 T_{c}$ for $10^{3} \mathrm{MCS}$, and then reverted at temperatures between 0.6 and $1.5 T_{c}$. The phase diagrams for Ising system ${ }^{17)}$ and $\mathrm{Al}-\mathrm{Zn}$ binary alloys ${ }^{18,19)}$ are shown in Fig. 3. The ageing and reversion conditions are denoted by boxes and circles, respectively. From the atomic configuration at each time, the structure of the alloy was examined directly, or from the structure function, $S(k, t)$, obtained from the Fourier transform of the system.

The simulation was performed on the FACOM M382 and M780 systems at Kyoto University.

\section{Results and Discussion}

\section{Solution Treatment and Ageing}

For the experimental investigation of phase decomposition in binary alloys, samples are solution treated before ageing. In the pioneer work concerning the phase decomposition in Ising lattice, Kalos, Lebowitz, Marro, and their coworkers ${ }^{2,4)}$ examined the structure change during ageing just above the coexistence curve,

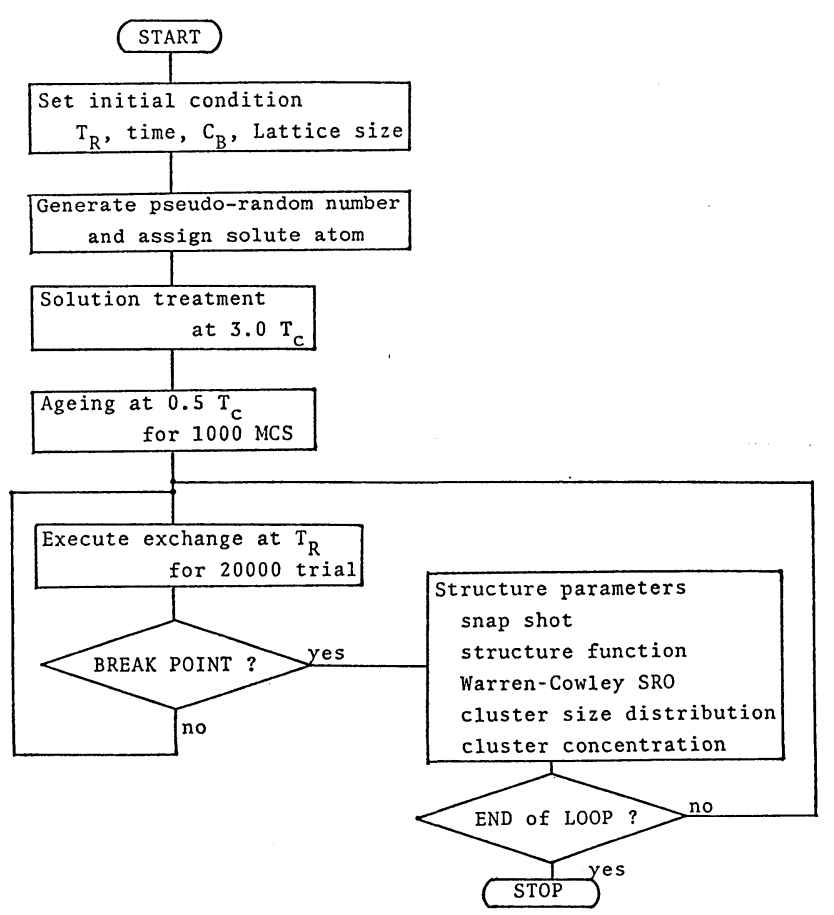

Fig. 2. General flow chart of the present simulation.
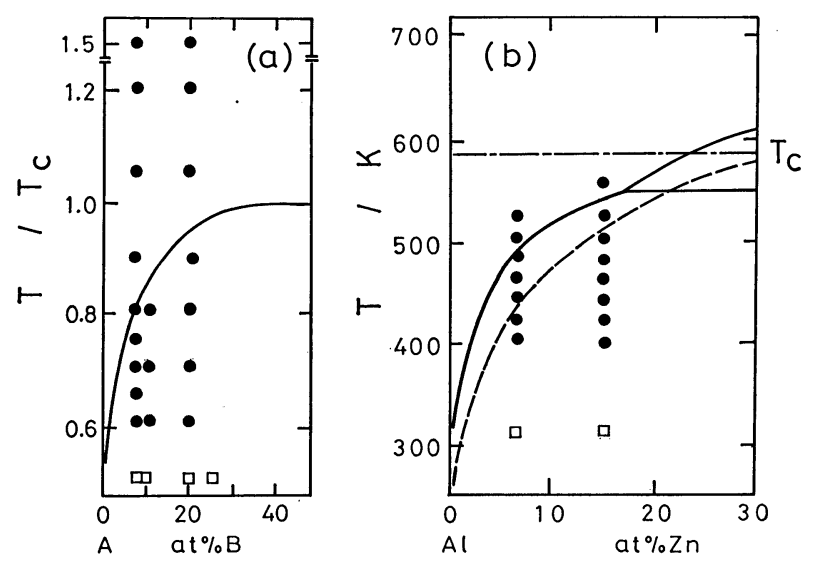

$\square$, $\bigcirc$ : The ageing and reversion temperatures, respectively.

Fig. 3. Phase diagrams for fcc Ising model (a) and $\mathrm{Al}-\mathrm{Zn}$ binary alloys (b).

and found that the structure function, $S(k, t)$, which is defined as the Fourier transform of the pair correlation function, $G(r, t)$ by the references ${ }^{1,4)}$ :

$$
S(k, t)=\sum_{\boldsymbol{r}} \exp (i \boldsymbol{k r}) G(\boldsymbol{r}, t)
$$

and

$$
G(r, t)=\mathcal{N}^{-1} \sum_{\boldsymbol{r}}\left\langle\left\{s\left(\boldsymbol{r}_{i}\right)-\bar{s}\right\}\left\{s\left(\boldsymbol{r}+\boldsymbol{r}_{i}\right)-\bar{s}\right\}\right\rangle,
$$

developed weakly with time, and converged to the envelope function given by Langer et al. ${ }^{20}$ ) The time evolution of the structure function during ageing at 1.2 and $2.0 T_{c}$ for $C_{B}=25 \%$ in the present study is shown in Fig. 4. It is seen that the change of $S(k, t)$ is qualitatively the same with the results by Lebowitz et. al., and $S(k, t)$ decreased as the ageing temperature increased. For the solution treatment condition 
adopted in the present simulation, i.e., $3.0 T_{c}$ for $C_{B}=$ 7.5 to $20 \%$, no clear development in the structure function was observed. However, Warren-Cowley short range order (SRO) calculated directly from the atomic configuration shows that the SRO for nearest neighbours remains some positive constant, even for

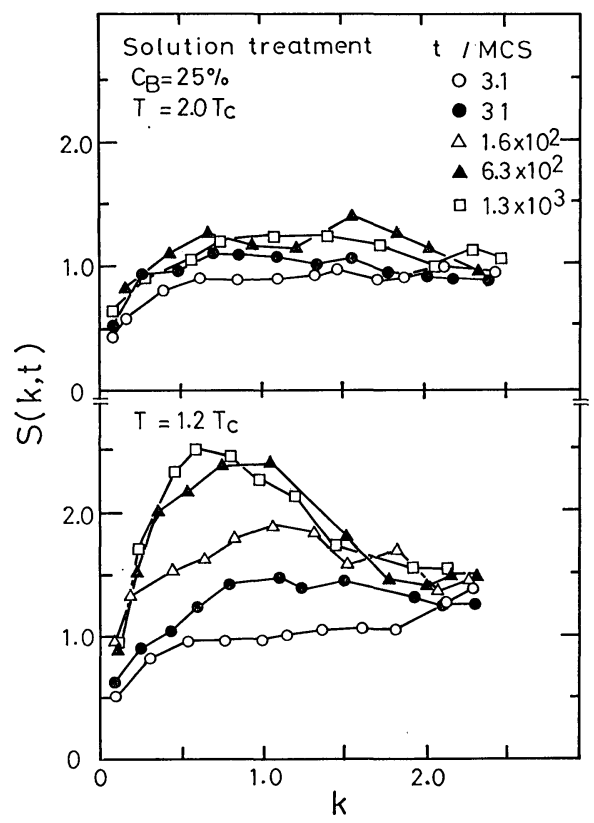

Fig. 4. Change of structure function during solution treatment above critical temperature, $T_{c}$. the present solution treatment condition, e.g., 0.0102 for $7.5 \%$ and 0.0249 for $20 \%$ at $3.0 T_{c}$, suggesting that the solute atoms tend to associate, even well above the coexistence curve. The structure change during ageing almost agreed with these obtained for the simple cubic system. For instance, the change of the scattering vector at the maximum $S(k, t), k_{m}$, agreed qualitatively with the one obtained by several authors..$^{5,6)}$ It is concluded that although the solution treatment can affect the kinetics of clustering for the very early stage of phase decomposition as denoted by Penrose et $a .^{10)}$ as 'first stage', no substantial deviation from the experimental or simulation results was found in the present simulation. On the basis of the phase-decomposed structure obtained by the procedure mentioned above, the structure change during reversion was investigated.

\section{Reversion above the Coexistence Curve}

When the pre-aged system containing various sizes of precipitates is held at the temperatures above the coexistence curve, all the precipitates dissolve to vanish into the matrix. Figure 5 shows a set of 'snap shots' of the system with $C_{B}=20 \%$ as observed on (100) and (111) cross section during reversion at 1.2 $T_{c}$. As reversion proceeded, it is seen that the precipitates became more diffuse and dissolved into the matrix. Finally, the precipitates vanished completely at 500 MCS, but there still remains a tendency for association. In order to estimate the degree of dissolu- $\left(\begin{array}{lll}1 & 0 & 0\end{array}\right)$

(a) $T_{R}=1.2 T_{c}$ 15.6 MCS

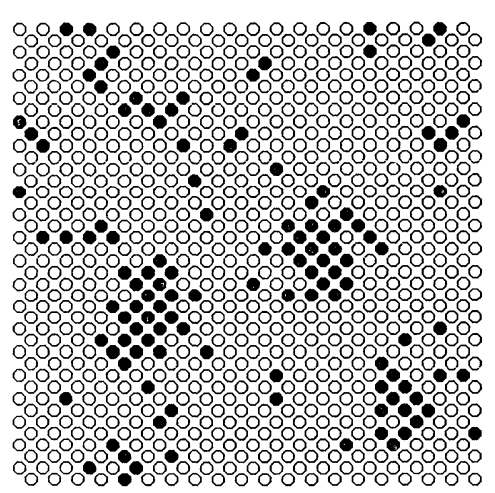

$\left(\begin{array}{lll}1 & 0 & 0\end{array}\right)$

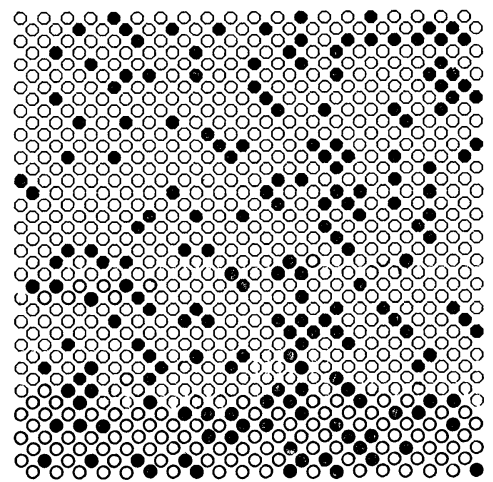

( $\left.\begin{array}{lll}1 & 1 & 1\end{array}\right)$

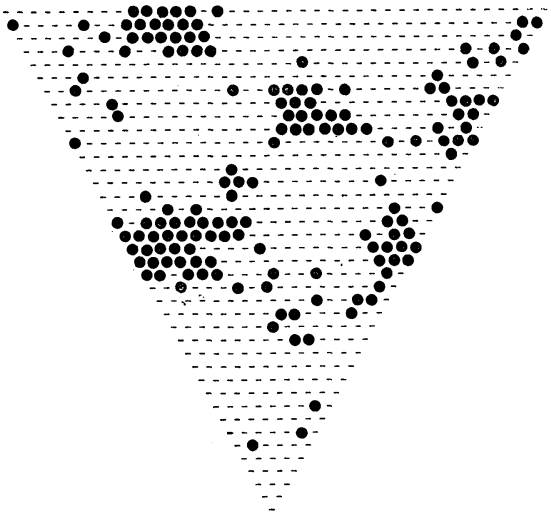

( $\left.\begin{array}{lll}1 & 1 & 1\end{array}\right)$

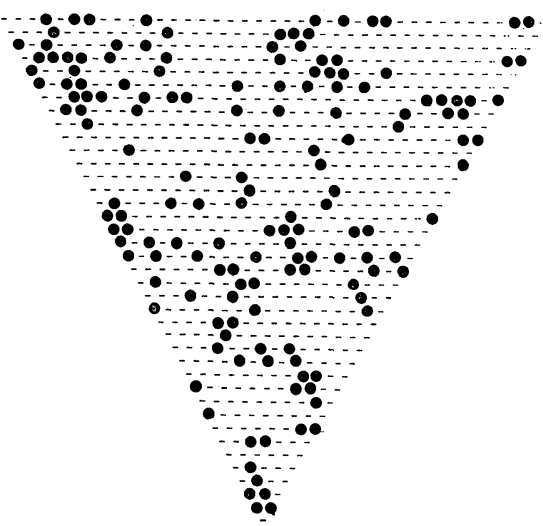

Fig. 5.

Snapshot of the system during reversion at $1.2 T_{c}$. Each figure represents the (111) and (100) cross section at (a) 15.6 and (b) 500 MCS.

(b) $T_{R}=1.2 T_{c}$ 500 MGS

:

$$
25
$$

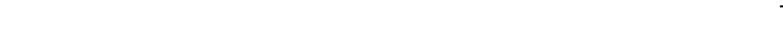

- 
tion of the precipitates during reversion quantitatively, the change in Warren-Cowley SRO was calculated directly from the atomic configuration according to the equation given by Warren. ${ }^{21}$ Figure 6 shows the change of SRO during reversion for $C_{B}=7.5 \%$ and $T_{R}=1.5 T_{c}$. The distance, $r$, in the figure corresponds to the distance for the SRO which is related to the neighbour sites denoted by the indices $(i, j, k)$ of fcc lattice by

$$
r=\sqrt{i^{2}+j^{2}+k^{2}} .
$$

It is seen that SRO decreased rapidly with time, and at $150 \mathrm{MCS}$, they converged to some constant values, which corresponded to the equilibrium value at the reversion temperature. The value for the nearest neighbour remained positive, and approached larger constant as the reversion temperature decreased at long reversion times. The order parameter monotonically decreased for $r<2.8$. However, for $r=3.6$, it is seen that the order parameter increased slightly from a negative to a positive value, and then gradually decreased to vanish. The change of SRO for $r=3.6$ can be explained as follows. For the preaged condition, the solute atoms associate to form precipitates which have sharp interfaces. This causes the positive value of SRO at small $r$. However, for the radial distance of slightly larger than the average diameter of the precipitates, SRO remains slightly negative. As reversion proceeds, solute atoms continue to flow out of the precipitates, which results in an increase in the solute concentration just outside the interface of precipitates, and then an increase in SRO. In this case, it is seen that SRO converged to zero at 150 MCS. It is concluded that the completion of reversion is estimated from the time evolution of SRO. In the following discussions, the change as shown above is analysed quantitatively from the structure parameters such as Warren-Cowley SRO, the average radii and cluster composition, $C_{p}$.

From the atomic configuration, the structure function was calculated according to Eqs. (1) and (2). The spherically averaged structure function, $S(k, t)$, corresponds to the small-angle scattering intensity from the polycrystalline sample, $I(k)$. The structure func-

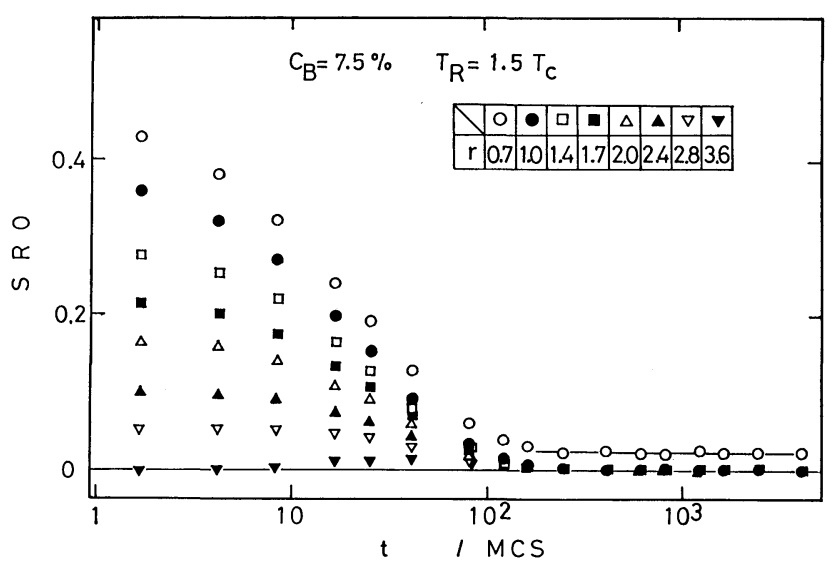

Fig. 6. Change of Warren-Cowley SRO for $C_{B}=7.5 \%$ reverted at $1.5 T_{c}$. tion during reversion at $1.5 T_{c}$ for $C_{B}=20 \%$ is shown in Fig. 7. Since the reversion temperature lies above the coexistence curve, it is seen that $S(k, t)$ decreased monotonically to vanish. The locus of the maximum of the structure function, $k_{m}$, remains almost constant at the early stage of reversion, and then shifted to the smaller $k$. This suggests that the average interparticle distance increased, and then the number of the precipitates decreased during reversion. ${ }^{13}$ ) The time evolution of the structure function during reversion for $C_{B}=7.5 \%$ and $T_{R}=0.9 T_{c}$ was also found to be qualitatively the same with the one at $1.5 \mathcal{T}_{c}$, as shown in Fig. 8. Change of the scattering intensity, $I(k)$, during reversion at $0.89 T_{c}$ was shown in Fig. 9 for $\mathrm{Al}-6.8 \mathrm{at} \% \mathrm{Zn}$, whose initial volume fraction is almost the same with the simulation for $C_{B}=7.5 \%$. It is seen that the change of the scattering intensity agreed well with the simulation.

In the SAXS experiment for $\mathrm{Al}-\mathrm{Zn}$ alloys, the average size of G.P. zone is determined from the scattering intensity in terms of Guinier approximation, ${ }^{22)}$ written as

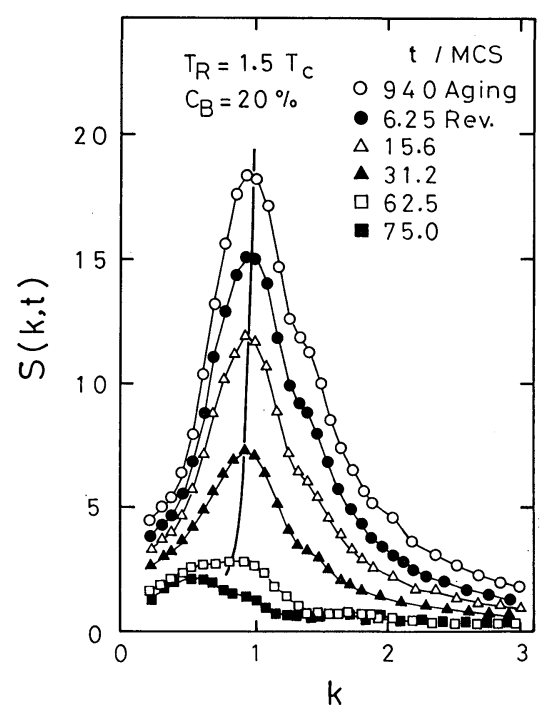

Fig. 7. Structure function for $C_{B}=20 \%$ during reversion at $1.5 T_{c}$.

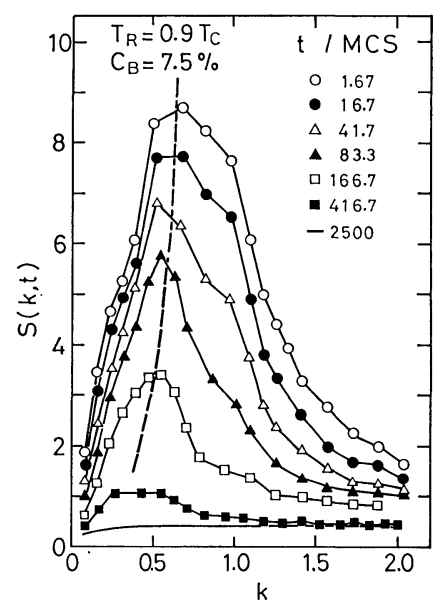

Fig. 8. Structure function for $C_{B}=7.5 \%$ during reversion at $0.9 T_{c}$. 


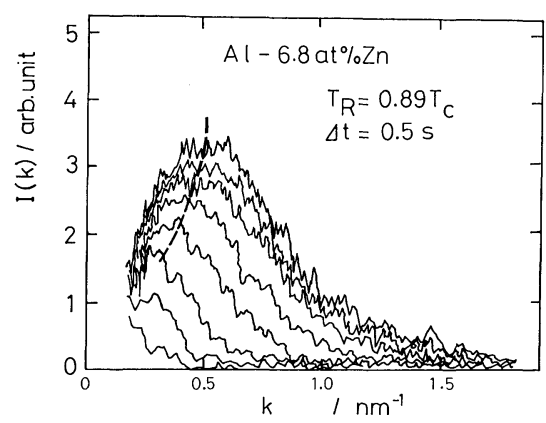

Fig. 9. Change of the scattering intensity for $\mathrm{Al}-6.8 \mathrm{at} \% \mathrm{Zn}$ reverted at $0.89 T_{c}$.

$$
I(k, t)=I_{0} \exp \left(-\left(k R_{G}\right)^{2} / 5\right),
$$

where, $R_{G}$ : Guinier size of the precipitates.

The time evolution of $R_{G}$ obtained experimentally for Al-15at $\% \mathrm{Zn}$ during reversion above the coexistence curve is shown in Fig. 10. For each reversion temperature, $R_{G}$ remains almost constant in the early stage, and then a rapid increase in size was observed. For the present simulation, the average radius can be also evaluated from the same equation simply replacing $I(k)$ by $S(k, t)$. Change of Guinier radius calculated for the structure function in reversion at $1.5 T_{c}$ is shown in Fig. 11, along with the cluster size obtained from the correlation function by using the same method as in the SAXS experiments. ${ }^{23)}$ It is seen that the time evolution of the cluster size is also very similar to the experimental results obtained by the SAXS experiments.

It has been made clear that the time evolution of the parameters which were obtained from the SAXS experiments are well described by the kinetic Ising model. Now the structure change of precipitates will be discussed in comparison with this model. As shown in Fig. 1, the structural change of individual G.P. zones for $\mathrm{Al}-\mathrm{Zn}$ was discussed on the assumption of two-phase model, and it was concluded ${ }^{13)}$ that the structure change during reversion above the coexistence curve and below $T_{c}$ was characterized by the early stage of decreasing zone composition and the later stage of shrinking zones. For the same reversion condition, the structure change of individual precipitates in the fcc Ising system was examined. Figure 12 shows the structure change of single spherical particles during reversion at $0.8 T_{c}$. The solute concentration, $C_{p}$, and the volume, $V_{p}$, of the particle, were calculated directly from the atomic configuration according to the criterion described elsewhere. ${ }^{24)}$ For the statistical stability, the solute concentration was calcuated only when the particle contained more than 100 atoms. It is seen that, as the initial radius of the particle increased, the whole process slowed down. The solute concentration of the particle decreased while its volume remained almost constant in the early stage. In the later stage, i.e., after about $200 \mathrm{MCS}$ for initial radius $R_{0}=6$ and $100 \mathrm{MCS}$ for $R_{0}=4.5$, the solute concentration converges to a constant of about $94 \%$. The arrows in the figure denote the time when the volume of the particle decreased by $5 \%$.

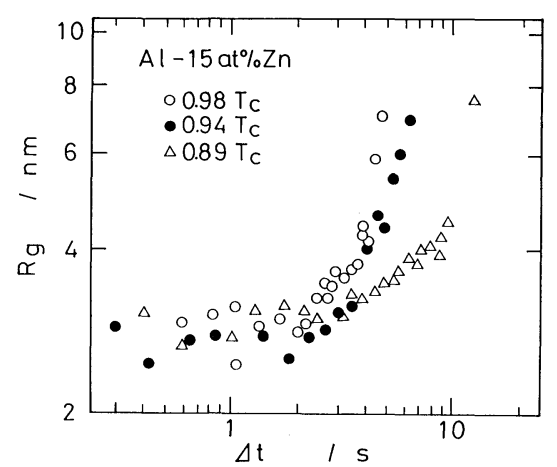

Fig. 10. Guinier radius for $\mathrm{Al}-15 \mathrm{at} \% \mathrm{Zn}$ during reversion as a function of time.

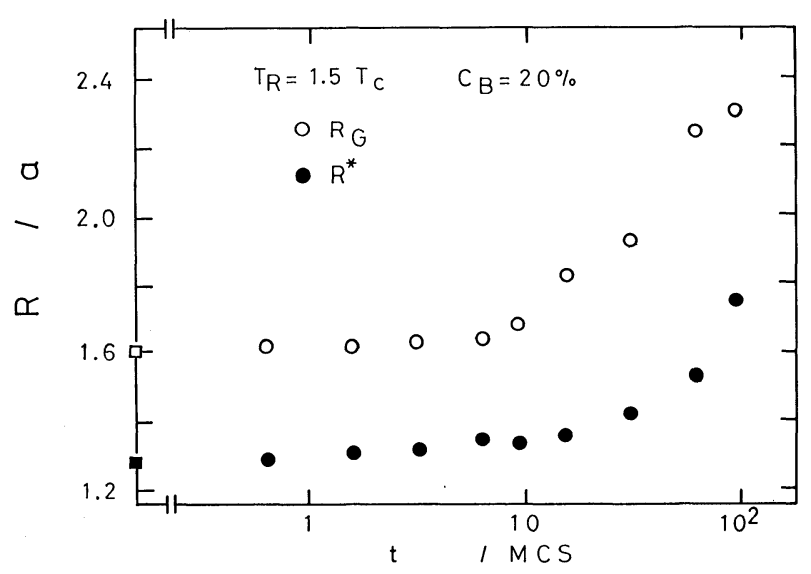

Fig. 11. Guinier radius for $C_{B}=20 \%$ during reversion at $1.5 T_{c}$.

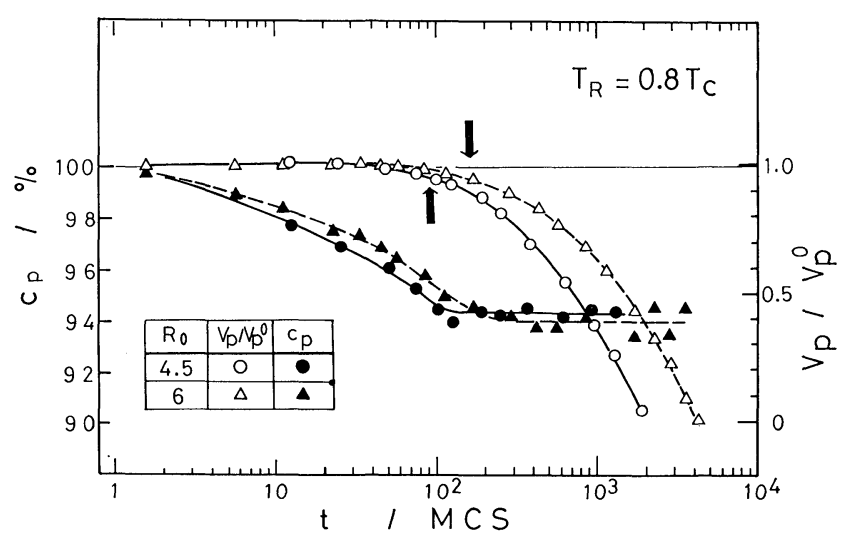

Fig. 12. Relative change of the volume and the solute concentration for single spherical precipitates during reversion at $0.8 T_{c}$.

The time agrees well with the stage when the concentration of the particle converged. Because the reversion temperature was above the coexistence curve, the particle dissolved completely in the later stage.

From the above results, it is concluded that the structure change during reversion above the coexistence curve for fcc Ising system as observed from the structure function and the Guinier radius agreed well with the SAXS measurements for Al- $\mathrm{Zn}$ binary alloys, and the structure change of individual particles has two distinct stages. This proved the validity of the two-phase assumption applied to the SAXS experi- 
ments.

\section{Reversion below the Coexistence Curve}

The reversion phenomena were also observed when the precipitates formed by ageing at $0.5 T_{c}$ were reverted below the coexistence curve. Figure 13 shows an example of the time evolution of the structure function for $C_{B}=20 \%$ reverted at $0.8 T_{c}$. Similar to the reversion above the coexistence curve, the structure function decreased at the first step. Subsequently, the structure function began to increase after 230 MCS, which is the most characteristic feature for the reversion below the coexistence curve, and which corresponds to the coarsening process at the reversion temperature. ${ }^{24)}$ This behaviour was observed also in the SAXS experiments for $\mathrm{Al}-15 \mathrm{at} \% \mathrm{Zn} .{ }^{25)} \quad$ Figure 14 shows the time evolution of the small-angle scattering intensity during reversion whose temperature and initial volume fraction of the precipitates corresponded
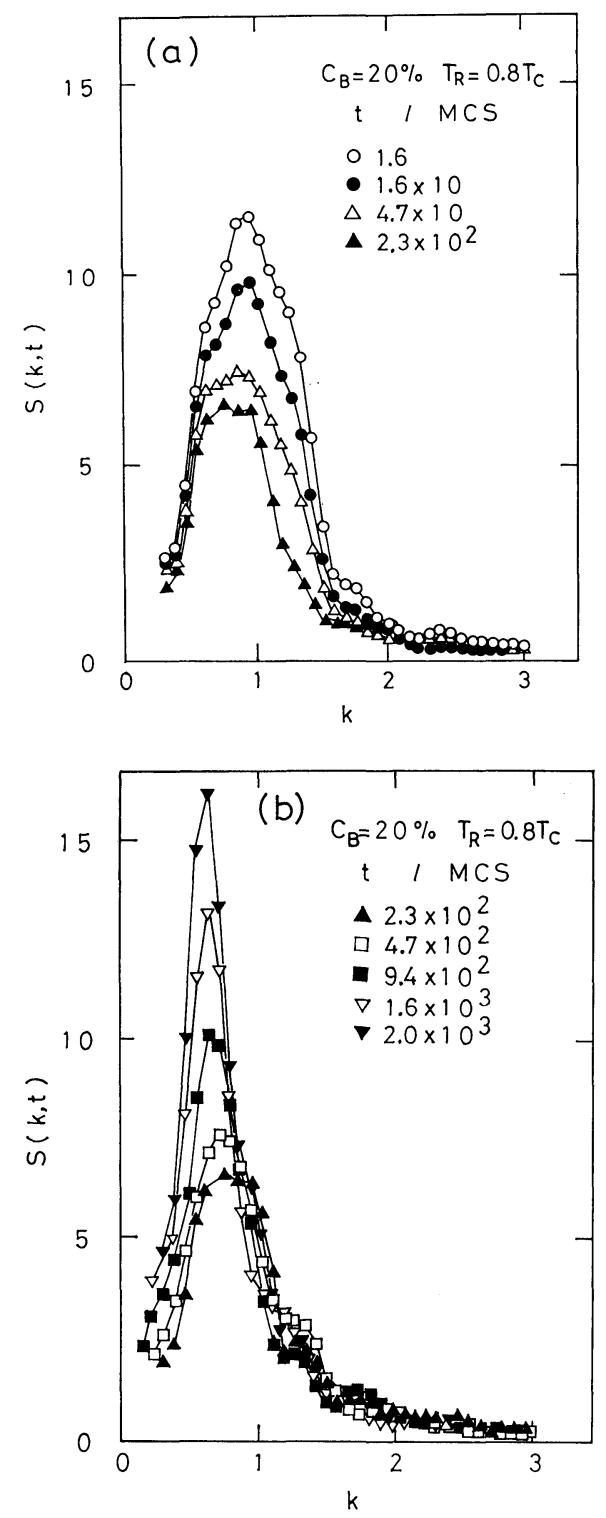

Fig. 13. Structure function for $C_{B}=20 \%$ during reversion at $0.8 T_{c}$, which is below the coexistence curve. Structure function decreased in the first step (a), and then began to increase (b). to the simulation condition of Fig. 12. It is seen that the change of the scattering intensity agreed with the simulation very well.

In the discussion on the change of SRO above the coexistence curve, it was found that the SRO for the nearest neighbour converged to a small positive constant during reversion and it could be used as a completion time of reversion. Change of SRO for $C_{B}=$ $7.5 \%$ and $T_{R}=0.6 T_{c}$ is shown in Fig. 15. The composition is the same with Fig. 6, but the temperature was below the coexistence curve here. It is seen that SROs for each radial distance decreased slowly with time firstly. The fact that the order parameters corresponding to a rather large radial distance as well as the first nearest neighbour remained positive means that a certain amount of clusters still existed even at the minimal point of the SRO at $t=8.3 \times 10^{2} \mathrm{MCS}$. Beyond about $1 \times 10^{3} \mathrm{MCS}$, the order parameters began to increase, suggesting that the solute atoms began to associate again. Thus, for the reversion below the coexistence curve, the onset of the increase in the SRO for the first nearest neighbour can be used as the onset time of coarsening, i.e., the completion of reversion at the temperature. Change of the SRO for the nearest neighbour site during reversion is shown

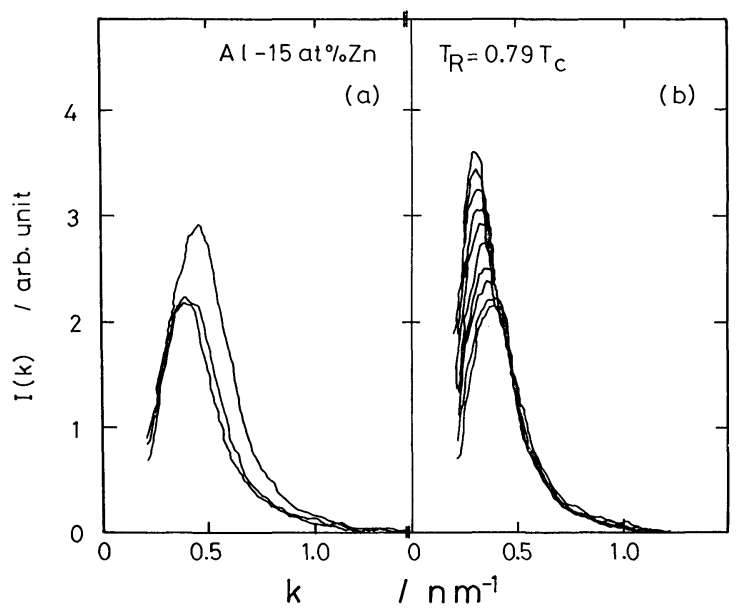

Fig. 14. Change of the scattering intensity for $\mathrm{Al}-15 \mathrm{at} \% \mathrm{Zn}$ during reversion at $0.8 T_{c}$. The scattering intensity decreased first (a), and then began to increase (b).

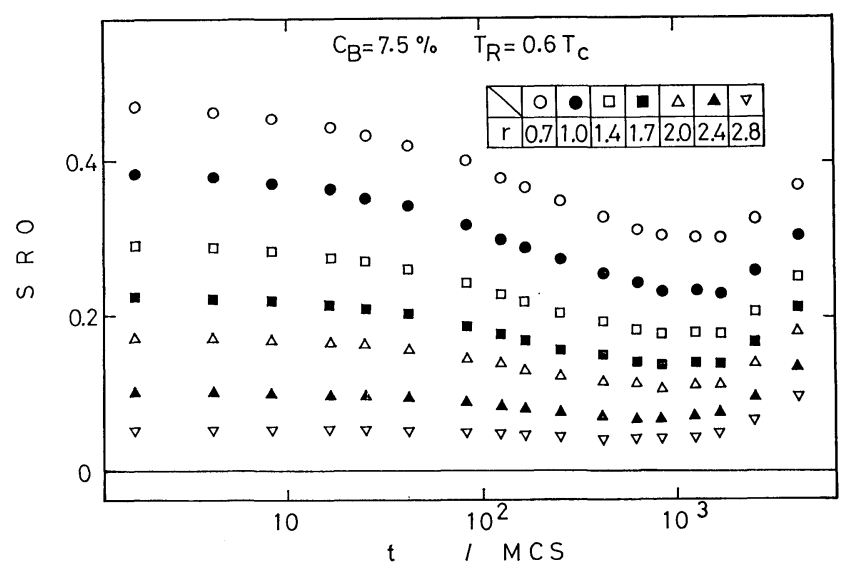

Fig. 15. Change of Warren-Cowley SRO for $C_{B}=7.5 \%$ during reversion at $0.6 T_{c}$. 
in Fig. 16 for $C_{B}=20 \%$. The decrease of the order parameter became more remarkable as the reversion temperature increased, and the onset of coarsening process which was shown by arrows in the figure delayed for the higher reversion temperatures. These onset times were found to agree well with the time when the structure function began to increase at each reversion temperature. For the same reversion temperature, the onset of the coarsening process was delayed as the alloy composition decreased. This is reasonable because the interparticle distance increases as the alloy composition decreases, and thus a longer time is necessary for diffusion.

From the results obtained for the structure function and SRO, the structure changes at the temperatures below the coexistence curve were clearly divided into reversion process and coarsening process. As the next step, the structure change during the reversion process was examined. Change in the composition of the precipitates, $\Delta c_{p}$, and in the Guinier radius are shown in Fig. 17. The composition of the particles was calculated only for those which contained more than 200 atoms, in order to suppress the effect of statistical error. It is seen that $\Delta c_{p}$ decreased in the early

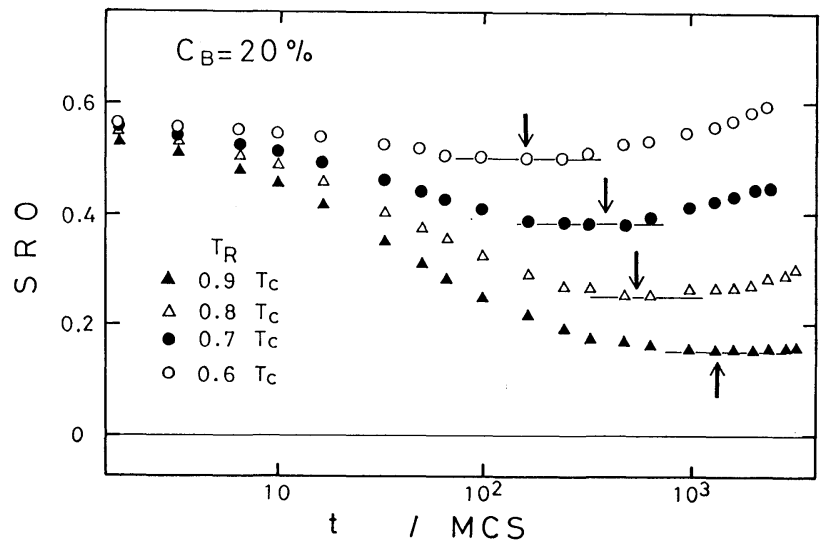

1 : The onset of coarsening process

Fig. 16. Change of the Warren-Cowley SRO for the nearest neighbour obtained for $C_{B}=20 \%$.

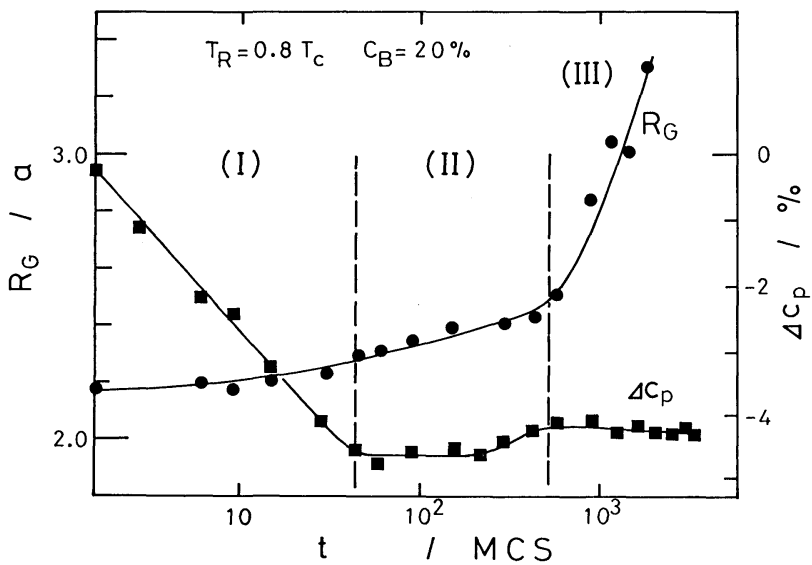

The boundaries in the figure:

The onset of the later stage (I/II) and coarsening (II/III).

Fig. 17. Change of the average concentration and the Guinier radius for $C_{B}=20 \%$ during reversion at $0.8 T_{c}$. stage of reversion and remained almost constant after 39.1 MCS. The reversion process can be divided into two stages in terms of the time when the composition of the precipitates reached a constant value. The early stage of reversion (denoted as (I) in the figure) is characterized by the compositional reduction of the precipitates. In the later stage of reversion (denoted as (II) in the figure) each precipitate began to shrink as shown in Fig. 12. For the closer examination, the composition increased slightly during the later stage of reversion. This behaviour is qualitatively explained by Gibbs-Thomson effect, because all the precipitates shrank during the stage.

During reversion, the Guinier radius continued to increase slightly, and then increase rapidly in the coarsening process. Two reasons can be considered for the increase of $R_{G}$ during reversion. One is that each precipitate forms a concentration profile just outside its interface, causing an apparent increase in the Guinier radius. This assumption is supported by the fact that SRO corresponding almost to the average diameter turned to a positive value temporarily during reversion. Another explanation is that, although all the precipitates shrink during reversion, the average size of 'surviving' precipitates increases because the precipitates dissolve faster as their initial radii are smaller, as shown in Fig. 12. Although these two effects could not be separated in the present simulation, the time evolution of SRO at a larger radial distance suggested that the former effect became dominant as the reversion temperature increased.

In the SAXS experiments, the changes of the solute concentration inside G.P. zones and the volume fraction were estimated by using two-phase model. ${ }^{13,25)}$ Relative change of the volume fraction and electron density difference are shown in Fig. 18 along with the boundary of reversion and coarsening determined experimentally for $\mathrm{Al}-15 \mathrm{at} \% \mathrm{Zn}$ reverted at $0.76 \mathcal{T}_{c}$. The electron density difference, $\Delta \rho$, is a parameter which is proportional to the compositional difference between the G.P. zone and the matrix. It is seen that the composition of G.P. zones decreased in the early

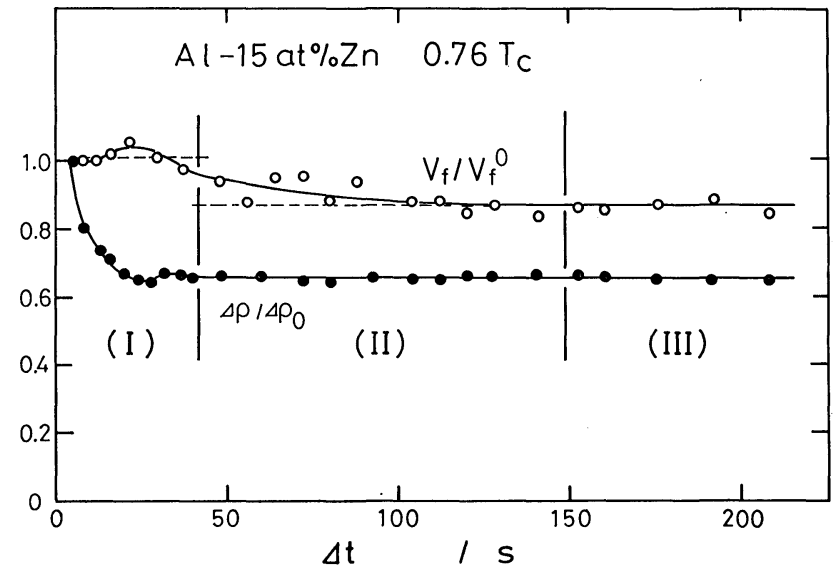

Fig. 18. Relative change of the solute concentration difference between G. P. zones and matrix, $\Delta \rho / \Delta \rho_{0}$, and the volume fraction for $\mathrm{Al}-15 \mathrm{at} \% \mathrm{Zn}$ during reversion at $0.76 T_{c}$, calculated on the assumption of two-phase model. 
stage, and then the volume fraction decreased during reversion. The onset of coarsening is shown by an arrow in the figure. During early stage of reversion, the volume fraction of G.P. zones exceeded unity slightly. This was explained as follows. ${ }^{13)}$ During dissolution, each G.P. zone forms a concentration gradient outside its zone/matrix interface. The apparent radius as observed by means of small-angle scattering increases due to the gradient, although the radius as defined from the locus of the interface remains the same. The apparent increase in the volume fraction was attributed to this apparent increase in the radius. This effect was estimated to be less important in the two phase analysis, ${ }^{13)}$ as confirmed in the present simulation.

From the above results and discussions, the structure change during reversion in fcc Ising system is divided into two stages in terms of SRO and the average concentration of the precipitates. Individual precipitates have their own time scale during reversion, according to the initial size. However, as a whole, it was found out that the average structure change is also divided clearly into two stages during reversion on the basis of the average structure parameters. This result comes from the fact that only the larger precipitates can affect the average structure parameters dominantly.

In the present simulation, it has been made clear that the structure change during reversion in fcc Ising system is a good description of that for $\mathrm{Al}-\mathrm{Zn}$ binary alloys. For the further comparison in time scale, the time prefactor in Eq. (1) should be correctly estimated. For the phase decomposition in a simple cubic lattice, Penrose et al. ${ }^{10)}$ commented that the simulation can be readily adjusted to the real time scale to the system such as $\mathrm{Al}-\mathrm{Zn}$ alloys. However, there are some problems to be examined in order to apply the simulation results to the phase decomposition of $\mathrm{Al}-\mathrm{Zn}$ alloys. One is the effect of vacancies. It is well known that the phase decomposition in quenched $\mathrm{Al}-\mathrm{Zn}$ alloys proceeds by quenched-in excess vacancies, whose concentration is not determined by the ageing temperature. Although Sur et $a{ }^{5}{ }^{5}$ ) have found that the kinetics of phase decomposition is not affected by the introduction of a constant concentration of vacancies, more detailed information will be required to compare with experimental results. In contrast, excess vacancies in the samples of the metastable (stage III $^{26)}$ ) state have already depleted, so that the reversion process is expected to be under equilibrium and thus a constant concentration of vacancies. The time charts for the onset of later stage of reversion and coarsening process are shown in Fig. 19 for $\mathrm{Al}-15 \mathrm{at} \% \mathrm{Zn}$ alloy and Ising system whose composition is $20 \%$. It should be noted that the initial volume fraction is the same for both systems. For Ising system, the time prefactor, $\tau$, is evaluated by

$$
\tau^{-1}=D_{0} \exp (-E / R T),
$$

where, $R$ : the gas constant

$E$ : the activation energy, $E=1 \times 10^{4} R$ in the present calculation.

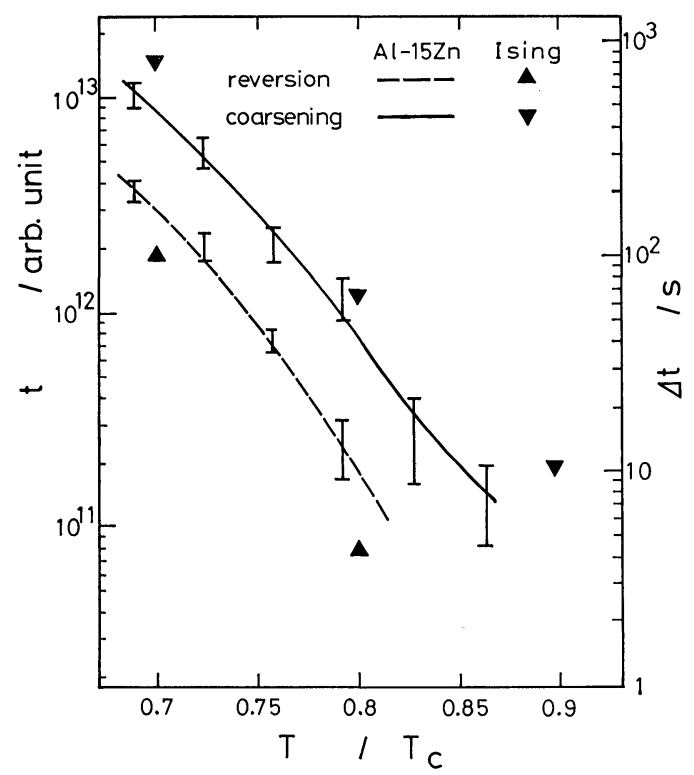

Fig. 19. Time-temperature chart for the onset of later stage of reversion and coarsening in $\mathrm{Al}-15 \mathrm{at} \% \mathrm{Zn}$ alloys and the present simulation.

It is seen that the time scale in the simulation, $t$, is adjustable to the experimental time scale, $\Delta t$, as

$$
t=2 \times 10^{10} \Delta t
$$

for a wide range of temperatures.

\section{Conclusion}

The structure change of a phase-decomposed binary fcc alloy during reversion has been investigated by using the kinetic Ising model. The results were compared with the experimental results obtained from SR-SAXS measurements for $\mathrm{Al}-\mathrm{Zn}$ alloys. It is made clear that:

(1) The reversion process has two distinct stages, regardless of whether the reversion temperature is above or below the coexistence curve. The early stage is characterized by a concentration reduction within the precipitates, and the later one is by a reduction of the precipitate volume fraction with constant concentration.

(2) For the reversion below the coexistence curve, the coarsening process was observed to follow the reversion process.

(3) The time evolution of structure paramenters during reversion for Ising system agreed well with those obtained by SR-SAXS measurements for the reversion of G.P. zones in $\mathrm{Al}-\mathrm{Zn}$ binary alloys. Furthermore, the structure change as observed in real space supported the two-phase interpretation applied to the $\mathrm{Al}-\mathrm{Zn}$ binary alloys.

(4) The time scale in the simulation (MSG) could be converted into the real time for $\mathrm{Al}-\mathrm{Zn}$ binary alloys for a wide range of temperatures. This suggested that the whole kinetics of reversion in the alloys was successfully described in the present simulation.

\section{REFERENCES}

1) A. Bortz, M. Kalos, J. Lebowitz and M. Zendejas: Phys. 
Rev., B10 (1974), 535.

2) M. Rao, M. Kalos, J. Lebowitz and J. Marro: Phys. Rev., B15 (1975), 4328.

3) M. Phani, J. Lebowitz, M. Kalos and O. Penrose: Phys. Rev. Lett., 45 (1980), 366.

4) J. Marro, J. Lebowitz and M. Kalos: Phys. Rev. Lett., 43 (1979), 282.

5) A. Sur. J. Lebowitz, J. Marro and M. Kalos: Phys. Rev., B15 (1977), 3014.

6) J. Lebowitz, J. Marro and M. Kalos: Acta metall., 30 (1982), 297.

7) P. Frazl, J. Lebowitz, J. Marro and M. Kalos: Acta metall., 31 (1983), 1849.

8) J. Marro and R. Toral: Physica, 122A (1983), 563.

9) M. Kalos, J. Lebowitz, O. Penrose and A. Sur: J. Stat. Phys., 18 (1978), 39.

10) O. Penrose, J. Lebowitz, J. Marro, M. Kalos and J. Tobochnik: J. Stat. Phys., 34 (1984), 399.

11) H. Ikeda and H. Matsuda: J. Jpn. Inst. Met., 48 (1984), 8.

12) K. Osamura, H. Okuda, H. Hashizume and Y. Amemiya: Acta metall., 33 (1985), 2199.

13) H. Okuda, K. Osamura, H. Hashizume and Y. Amemiya: Acta metall., 36 (1988), 899.

14) K. Kawasaki: Phase Transition and Critical Phenomena, Vol. 2, ed. Domb and Green, Academic Press, New York,
(1972), 443.

15) K. Binder: Monte Carlo Methods in Statistical Physics, 2nd Ed., ed. by K. Binder, Springer Verlag, Berlin, (1986), 1.

16) K. Binder, M. Kalos, J. Lebowitz and J. Marro: $A d v$. Colloid Interface Sci., 10 (1979), 173.

17) J. W. Eassam and M. E. Fisher: J. Chem. Phys., 38 (1963), 802.

18) T. Sato and Y. Kojima: Bull. Jpn. Inst. Met., 48 (1979), 669.

19) K. Satyanarayana: J. Mater. Sci., 16 (1981), 1233.

20) J. S. Langer, M. Bar-on and H. Miller: Phys. Rev., A11 (1975), 1417.

21) B. Warren: X-ray Diffraction, Addison-Wesley, New York, (1969).

22) A. Guinier and G. Fournet: Small-Angle Scattering of Xrays, John Wiley and Sons, New York, (1955).

23) K. Osamura and Y. Murakami: J. Jpn. Inst. Met., 43 (1979), 537.

24) H. Okuda and K. Osamura: Trans. Jpn. Inst. Met., 29 (1988), 198.

25) K. Osamura, S. Ochiai, H. Okuda, H. Hashizume and Y. Amemiya: Photon Factory Activity Report, 4 (1986), 317.

26) K. Osamura, H. Okuda and S. Ochiai: Scr. metall., 19 (1985), 1379. 